\title{
MARCO BRUNELLA
}

\section{On the discrete Godbillon-Vey invariant and Dehn surgery on geodesic flows}

Annales de la faculté des sciences de Toulouse $6^{e}$ série, tome $3, \mathrm{n}^{\circ} 3$ (1994), p. 335-344

<http://www.numdam.org/item?id=AFST_1994_6_3_3_335_0>

(C) Université Paul Sabatier, 1994, tous droits réservés.

L'accès aux archives de la revue «Annales de la faculté des sciences de Toulouse » (http://picard.ups-tlse.fr/ annales/) implique l'accord avec les conditions générales d'utilisation (http://www.numdam.org/conditions). Toute utilisation commerciale ou impression systématique est constitutive d'une infraction pénale. Toute copie ou impression de ce fichier doit contenir la présente mention de copyright.

\section{Numdam}

Article numérisé dans le cadre du programme

Numérisation de documents anciens mathématiques

http://www.numdam.org/ 


\title{
On the discrete Godbillon-Vey invariant and Dehn surgery on geodesic flows ${ }^{(*)}$
}

\author{
Marco Brunella ${ }^{(1)}$
}

RÉSUMÉ. - On donne une méthode pour construire des surfaces sectionnelles pour le flot géodésique d'une surface fermée à courbure négative, et dont l'application de premier retour est semi-conjuguée à un difféomorphisme torique Anosov. Cette construction est utilisée pour montrer que l'invariant discret de Godbillon-Vey [GS] n'est pas un invariant topologique, même quand on le restreint à $P L_{+}^{\lambda}\left(\mathrm{S}^{1}\right)$. Une variation de cette construction permet de construire des exemples de flots d'Anosov transitif liés à $[\mathrm{BL}]$.

Abstract. - We give a method to construct surfaces of section for the geodisic flow on a negatively curved closed surface, with first return map semiconjugate to a toral Anosov diffeomorphism. This construction is used to prove that the discrete Godbillon - Vey invariant [GS] is not a topological invariant, even when restricted to $P L_{+}^{\lambda}\left(\mathbf{S}^{1}\right)$. A variation on that construction produces examples of transitive Anosow flows related to $[\mathrm{BL}]$.

\section{Introduction}

Let $\mathrm{PL}_{+}\left(\mathbf{S}^{1}\right)$ be the group of orientation preserving piecewise linear homeomorphisms of the circle $\mathbf{S}^{1}=\mathbf{R} / \mathbb{Z}$. The discrete Godbillon-Vey class [GS] is the class $\overline{G V} \in H^{2}\left(\mathrm{PL}_{+}\left(\mathrm{S}^{1}\right), \mathbb{R}\right)$ represented by the 2-cocycle

$$
(f, g) \mapsto \frac{1}{2} \sum_{x \in \mathbf{S}^{1}} \operatorname{det}\left(\begin{array}{cc}
\log g^{\prime}(x+) & \log (f \circ g)^{\prime}(x+) \\
\log \left(g^{\prime}(x+) / g^{\prime}(x-)\right) & \log \left((f \circ g)^{\prime}(x+) /(f \circ g)^{\prime}(x-)\right)
\end{array}\right)
$$

(*) Reçu le 21 octobre 1993

(1) Dipartimento di Matematica, Piazza di Porta S. Donato 5, Bologna 
where, if $h \in \mathrm{PL}_{+}\left(\mathbf{S}^{1}\right), h^{\prime}(x+)\left(h^{\prime}(x-)\right)$ denotes the right (left) derivative of $h$ at the point $x \in \mathbf{S}^{1}$.

If $\Phi: G \rightarrow \mathrm{PL}_{+}\left(\mathrm{S}^{1}\right)$ is a representation of a group $G$ into $\mathrm{PL}_{+}\left(\mathbf{S}^{1}\right)$, we can set

$$
\overline{\mathrm{GV}}(\Phi)=\Phi^{*}(\overline{\mathrm{GV}}) \in H^{2}(G, \mathbb{R}),
$$

where $\Phi^{*}: H^{*}\left(\mathbf{P L}_{+}\left(\mathbf{S}^{1}\right), \mathbb{R}\right) \rightarrow H^{*}(G, \mathbb{R})$ is the map induced by $\Phi$. In particular, if $G$ is the fundamental group of a closed oriented surface $\Sigma_{g}$ of positive genus $g$, then we can identify $\overline{\mathrm{GV}}(\Phi)$ with the real number obtained from the canonical isomorphisms $H^{2}\left(\pi_{1}\left(\Sigma_{g}\right), \mathbb{R}\right) \simeq H^{2}\left(\Sigma_{g}, \mathbb{R}\right) \simeq \mathbb{R}$. This real number is the discrete Godbillon-Vey invariant of the representation $\Phi: \pi_{1}\left(\Sigma_{g}\right) \rightarrow \mathrm{PL}_{+}\left(\mathbf{S}^{1}\right)$.

Let $\lambda \in \mathbb{R}^{+}$and let $\mathbf{P L}_{+}^{\lambda}\left(\mathbf{S}^{1}\right)$ be the subgroup of $\mathrm{PL}_{+}\left(\mathbf{S}^{1}\right)$ composed by homeomorphisms $h$ such that $h^{\prime}(x) \in\left\{\lambda^{n} \mid n \in \mathbb{Z}\right\}$ at every differentiable point. The discrete Godbillon-Vey invariant of a representation $\Phi$ : $\pi_{1}\left(\Sigma_{g}\right) \rightarrow \mathrm{PL}_{+}^{\lambda}\left(\mathbf{S}^{1}\right)$ is then an integral multiple of $(1 / 2)(\log \lambda)^{2}$. In [Ghy] (see also $[\mathrm{Ha} 2],[\mathrm{HM}])$, E. Ghys constructs two representations

$$
\Phi_{j}: \pi_{1}\left(\Sigma_{g}\right) \rightarrow \mathbf{P L}_{+}^{\lambda_{j}}\left(\mathbf{S}^{1}\right), \quad j=1,2,
$$

which are topologically conjugate but which have different Godbillon-Vey invariant; the proof of this second property is based on the fact that $\lambda_{1}$ and $\lambda_{2}$ are different so that

$$
\frac{\left(\log \lambda_{1}\right)^{2}}{\left(\log \lambda_{2}\right)^{2}} \notin \mathbb{Q} \text {. }
$$

The aim of this note is to prove that even restricted to $\operatorname{PL}_{+}^{\lambda}\left(\mathbf{S}^{1}\right)$ the discrete Godbillon-Vey invariant is not a topological invariant, at least for some $\lambda$.

THEOREM . - There exist $\lambda \in \mathbb{R}^{+}$and two representations

$$
\Phi_{j}: \pi_{1}\left(\Sigma_{409}\right) \rightarrow \mathrm{PL}_{+}^{\lambda}\left(\mathbf{S}^{1}\right), \quad j=1,2
$$

which are topologically conjugate and which have different discrete Godbillon-Vey invariant.

The proof of this result is strongly inspired from [Ghy]: our representations $\Phi_{j}$ are topologically conjugate to the representation (into Diff ${ }_{+}^{\infty}\left(\mathbf{S}^{1}\right)$ ) corresponding to the stable foliation of the geodesic flow on $\Sigma_{409}$ w.r. to an 
hyperbolic metric. The main step is the observation that the geodesic flow on an hyperbolic surface $\Sigma_{g}, g \geq 2$, admits two essentially different surfaces of section of genus one: the one introduced by Birkhoff ([Fri], [Ghy]) and another one which we describe in Section 2. We give a description of these surfaces of section which is a little different from that explained in [Fri]; this will lead to the digression of Section 3, where we construct transitive Anosov flows which admit a transverse torus which is not a global cross section (compare $[\mathrm{BL}]$ for other examples).

\section{The surface of section of Birkhoff}

Let $\Sigma_{g}$ denote a closed oriented surface of genus $g \geq 2$ equipped with any hyperbolic metric. Let $\phi_{t}: T_{1} \Sigma_{g} \rightarrow T_{1} \Sigma_{g}$ denote the (Anosov) geodesic flow on the unitary tangent bundle of $\Sigma_{g}$. The topological equivalence class of $\phi_{t}$ does not depend on the chosen hyperbolic metric.

Let $\left\{\gamma_{j}\right\}_{j=1}^{2 g+2}$ be the collection of simple closed oriented geodesics on $\Sigma_{g}$ shown in the following picture:

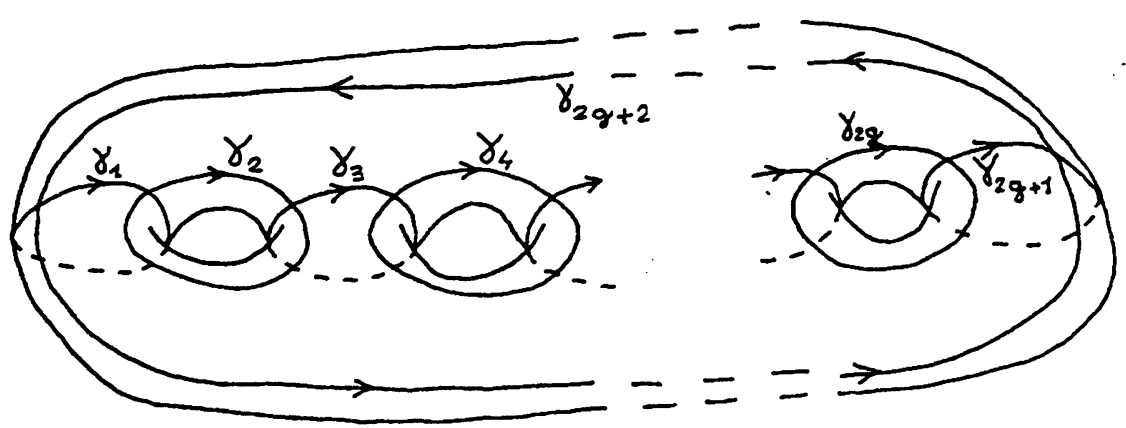

Fig. 1

The geodesics $\left\{ \pm \gamma_{j}\right\}_{j=1}^{2 g+2}\left(+\gamma_{j}=\gamma_{j},-\gamma_{j}=\gamma_{j}\right.$ with reversed orientation) lift to $T_{1} \Sigma_{g}$ to $4 g+4$ closed orbits of the flow $\phi_{t}$, which we will denote by $\left\{\Gamma_{j}^{ \pm}\right\}_{j=1}^{2 g+2}\left(\Gamma_{j}^{ \pm}\right.$projects onto $\left.\pm \gamma_{j}\right)$. These closed orbits are boundary of a surface of section $S$ for $\phi_{t}$ : $S$ is an embedded surface in $T_{1} \Sigma_{g}$, with $\partial S=\bigcup_{j=1}^{2 g+2} \Gamma_{j}^{ \pm}, \int S$ is transverse to $\phi_{t}$ and every orbit of $\phi_{t}$ intersects $S$ in a uniformly bounded time. This surface $S$ can be described in two ways.

Description 1 ([Fri]). $-\Sigma_{g} \backslash \bigcup_{j=1}^{2 g+2} \gamma_{j}$ is composed by four $(2 g+2)$-gons. Take two of these $(2 g+2)$-gons, such that their closures intersect only in 
correspondence of their vertices, and fill them with two foliations by strictly convex circles with a centre-type singularity. Then $S$ is the closure of the set of unitary tangent vectors which are tangent to these convex circles. It is easy to see that $S$ is a torus with $4 g+4$ holes.

Description 2. - Each $\gamma_{j}$ has a natural coorientation, induced by the orientations of $\Sigma_{g}$ and $\gamma_{j}$. For every $j=1, \ldots, 2 g+2$ let $C_{j}$ be the set of unitary tangent vectors at points of $\gamma_{j}$ which are non-negative w.r. to the coorientation of $\gamma_{j}$. Each $C_{j}$ is a closed cylinder, bounded by $\Gamma_{j}^{+}$and $\Gamma_{j}^{-}$; it intersects $C_{j-1}$ and $C_{j+1}$ along segments and does not intersect the other $C_{i}$ 's; $\int C_{j}$ is transverse to $\phi_{t}$. A surgery along $C_{j} \cap C_{j+1}$, as described in [Fri], produces an embedded surface $S^{\prime}$, bounded by $U_{j=1}^{2 g+2} \Gamma_{j}^{ \pm}$, diffeomorphic to the torus with $4 g+4$ holes. This $S^{\prime}$ is a surface of section for $\phi_{t}$.

The two surfaces $S$ and $S^{\prime}$ are isotopic rel(boundary), the isotopy being realized be the flow $\phi_{t}$ itself. The flow $\phi_{t}$ induces on $S$ (or $S^{\prime}$ ) a first return map $f$, which is semiconjugate to an hyperbolic toral automorphism ([Ghy], [Ha1]). The conjugacy class of the automorphism has been calculated by $\mathrm{N}$. Hashiguchi (see also [Chr] for a particular case and [Ghy] for the computation of the trace).

Proposition 1 ([Ha1]). - The first return map $f$ defined by $\phi_{t}$ on the surface of section $S$ is topologically semiconjugate to the hyperbolic toral automorphism defined by

$$
A_{g}=\left(\begin{array}{cc}
2 g^{2}-1 & 2 g^{2}+2 g \\
2 g^{2}-2 g & 2 g^{2}-1
\end{array}\right) .
$$

Let $\mathcal{F}^{s}$ be the stable foliation of $\phi_{t}$. It is transverse to the fibres of $T_{1} \Sigma_{g} \rightarrow \Sigma_{g}$ and hence it corresponds to a representation

$$
\Psi_{g}: \pi_{1}\left(\Sigma_{g}\right) \rightarrow \operatorname{Diff}_{+}^{\infty}\left(\mathbf{S}^{1}\right) .
$$

Let $\lambda_{g} \in(1,+\infty)$ be the largest eigenvalue of $A_{g}$. The above proposition, or more simply the computation of $\lambda_{g}$ done in [Ghy], has the following consequence.

COROLlaRY 1 ([Ghy], [Ha2]). - The representation $\mathbf{\Psi}_{g}$ is topologically conjugate to a representation

$$
\Phi_{g}: \pi_{1}\left(\Sigma_{g}\right) \rightarrow \mathbf{P L}_{+}^{\lambda_{g}}\left(\mathbf{S}^{1}\right) .
$$

The explicit form of $\Phi_{g}$ can be found in [Ha2]. 


\section{Another surface of section}

Let $\left\{\mu_{j}\right\}_{j=1}^{2 g+1}$ be the collection of simple closed oriented geodesics on $\Sigma_{g}$ shown in the following picture.

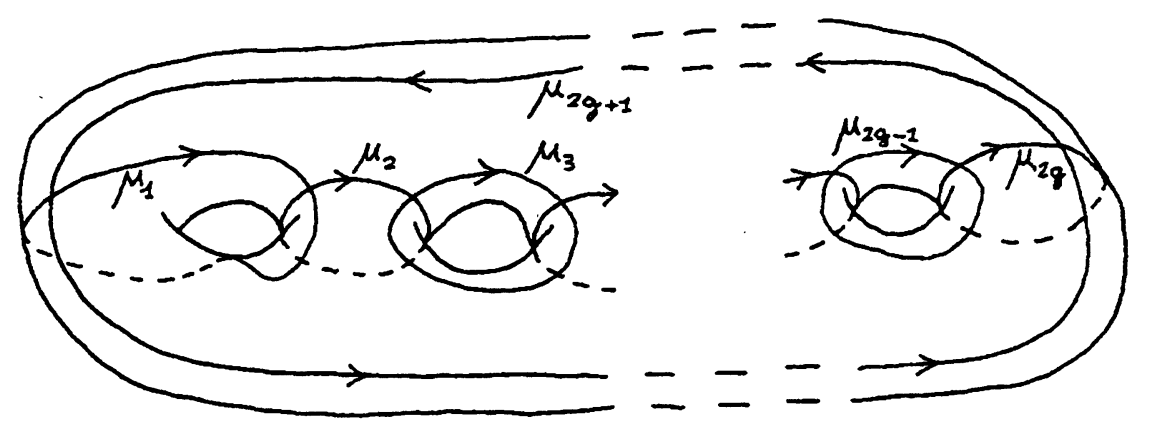

Fig. 2

(We have replaced $\gamma_{1}$ and $\gamma_{2}$ with a single geodesic $\mu_{1}$, freely homotopic to the product of $\gamma_{1}$ and $\gamma_{2}$.)

Let $\left\{M_{j}^{ \pm}\right\}_{j=1}^{2 g+1}$ be the closed orbits of $\phi_{t}$ corresponding to $\left\{ \pm \mu_{j}\right\}_{j=1}^{2 g+1}$. Then $\bigcup_{j=1}^{2 g+1} M_{j}^{ \pm}$is the boundary of a surface of section $T$ for $\phi_{t}$, which, as before, can be constructed in two ways.

Description 1. $-\Sigma_{g} \backslash \bigcup_{j=1}^{2 g+1} \mu_{j}$ is composed by two $(2 g+1)$-gons and one $(4 g+2)$-gon. The closures of the two $(2 g+1)$-gons intersect only at vertices, and the closure of the $(4 g+2)$-gon has selfintersections in correspondence of its vertices. We may repeat the construction of Section 1 starting either from foliations by convex circles on the two $(2 g+1)$-gons, or from a foliation on the single $(4 g+2)$-gon.

Description 2. - Same construction of Section 1, using cylinders over geodesics $\mu_{j}$.

These two constructions produce surfaces of section which are isotopic rel(boundary) and which are diffeomorphic to a torus with $4 g+2$ holes.

Working as in [Ha1] (see also [Chr], [Ghy]) one obtains the following analogue of Proposition 1. 
Proposition 1'. - The first return map $h$ defined by $\phi_{t}$ on the surface on section $T$ is topologically semiconjugate to the hyperbolic toral automorphism defined by

$$
B_{g}=\left(\begin{array}{cc}
4 g^{2}-2 g-1 & 2 g^{2}-2 g \\
8 g^{2}-2 & 4 g^{2}-2 g-1
\end{array}\right) .
$$

Let $\nu_{g} \in(1,+\infty)$ be the largest eigenvalue of $B_{g}$.

Corolla RY 1'. - The representation $\Psi_{g}$ is topologically conjugate to a representation

$$
\Phi_{g}^{\prime}: \pi_{1}\left(\Sigma_{g}\right) \rightarrow \mathrm{PL}_{+}^{\nu_{g}}\left(\mathbf{S}^{1}\right) \text {. }
$$

\section{Digression: Anosov flows with a transverse torus}

Let $\left\{\eta_{j}\right\}_{j=1}^{N}$ be a collection of closed oriented (hence cooriented) geodesics on $\Sigma_{g}$, not necessarily simple, such that:

(i) $\bigcup_{j} \eta_{j}$ has no triple point,

(ii) every other geodesic on $\Sigma_{g}$ intersects some $\eta_{j}$ in the positive direction.

For example, $\left\{\eta_{j}\right\}_{j}$ can reduce to a single "long" closed geodesic, "well distributed" in $\Sigma_{g}$.

Then construction 2 of the previous two sections (construction 1 is not always available) produces a surface of section $\Omega$ for $\phi_{t}$ with boundary $\bigcup_{j=1}^{N} N_{j}^{ \pm}$, where $N_{j}^{ \pm}$is the lift of $\pm \eta_{j}$.

It is not difficult to see that the stable foliation $\mathcal{F}^{s}$ induces on $\Omega$ a foliation $\mathcal{G}$ with $2 k_{j}^{ \pm}$semisaddles on $N_{j}^{ \pm}$, where $k_{j}^{ \pm}$is the number of positive intersections of $\pm \eta_{j}$ with $\bigcup_{i=1}^{N} \eta_{i}$. This permits the computation of the homeomorphism type of $\Omega$, via Poincaré-Hopf formula.

In particular, $\Omega$ has genus one and the first return map is topologically semiconjugate to an hyperbolic toral automorphism if and only if $k_{j}^{ \pm}=1$ for every $j$. This means that every $\eta_{j}$ is simple and that (modulo reordering) $\eta_{j} \cap \eta_{i}=\emptyset$ for $|j-i| \geq 2, \eta_{j+1}\left(\eta_{j-1}\right)$ intersects $\eta_{j}$ in exactly one point and in negative (positive) direction. This, in turn, implies that $\left\{\eta_{j}\right\}_{j}$ coincides with one of the collections $\left\{\gamma_{j}\right\}_{j=1}^{2 g+2}$ or $\left\{\mu_{j}\right\}_{j=1}^{2 g+1}$ previously examinated (remark that condition (ii) implies that $\Sigma_{g} \backslash \bigcup_{j} \eta_{j}$ is simply connected). 
For this reason, we don't know how to construct other surfaces of section with first return map semiconjugate to a hyperbolic toral automorphism, different from the two constructed above.

Because first return maps associated to surfaces of section are semiconjugate to pseudo Anosov diffeomorphism [Fri], our construction gives very concrete examples of such diffeomorphisms. The geodesics which form the boundary of the surface of section will correspond to fixed points of the pseudo Anosov diffeomorphism, and a geodesic which intersects $\bigcup_{j} \eta_{j}$ $k$ times in the positive direction will correspond to a periodic point of period $k$. For instance, if $\left\{\eta_{j}\right\}_{j}$ is a single long closed geodesic, then the corresponding first return map will be semiconjugate to a pseudo Anosov diffeomorphism on a surface of large genus whose singular set is composed by only two (fixed) points. A "generic" choice of the geodesic will ensure that such a diffeomorphism is not a branched covering of a toral automorphism.

Let now the collection $\left\{\eta_{j}\right\}_{j=1}^{N}$ of closed oriented geodesics satisfy:

(i) $\bigcup_{j} \eta_{j}$ is connected and has no triple point,

(ii) $k_{j}^{ \pm}=1$ for every $j$,

but doesn't satisfy the condition that every other geodesic intersects some $\eta_{j}$ in the positive direction. Then a Dehn surgery along $\left\{\eta_{j}\right\}_{j=1}^{N}[$ Fri] , [Goo] produces a transitive Anosov flow $\psi_{t}: M \rightarrow M$ which admits a transverse torus $T$ but which is not the suspension of a toral Anosov automorphism (compare $[\mathrm{BL}]$ ). For example, $\left\{\eta_{j}\right\}_{j}$ can be a single closed geodesic with exactly one point of self-intersection, or it can be a "piece" of the collection of Birkhoff's section (fig. 3).
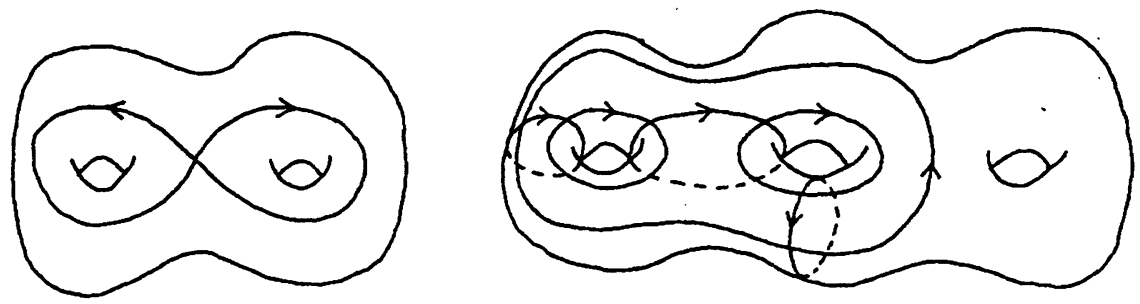

Fig. 3

Remark that, in general, the maximal $\psi_{t}$-invariant set in $M \backslash T$ will be large, i.e. not reduced to a finite collection of closed orbits (this is a difference with $[\mathrm{BL}]$ ). In the second example above, this maximal invariant set is isomorphic to the set of geodesics (lifted in $T_{1} \Sigma_{g}$ ) which are completely contained in an handle of $\Sigma_{g}$ (the handle to the right in the picture). If 
$\gamma \subset \Sigma_{g}$ is the unoriented geodesic which separates this handle from the region of $\Sigma_{g}$ containing $\bigcup_{j} \eta_{j}$ and if $T_{\gamma} \subset T_{1} \Sigma_{g}$ is its preimage in $T_{1} \Sigma_{g}$, then after the Dehn surgery $T_{\gamma}$ will correspond to a torus $S \subset M$ (disjoint from $T$ ) with the properties:

(a) $S$ contains two closed orbits $\gamma_{1}$ and $\gamma_{2}$ of $\psi_{t}$ and is transverse to $\psi_{t}$ outside $\gamma_{1} \cup \gamma_{2}$;

(b) $S$ separates $M$ in two pieces, one of which is a "piece" of geodesic flow (or the geodesic incomplete flow on a holed torus with negative curvature and geodesic boundary), the other one is diffeomorphic to the complement of a solid torus embedded in a torus bundle over the circle (more precisely, the torus bundle whose monodromy is $A_{2}$ ) and disjoint from a fiber (e.g. T) of that bundle.

Hence that example is a sort of connected sum between two classical examples of Anosov flows, or it is a suspension of a toral automorphism $\left(A_{2}\right)$ to which a handle (a piece of geodesic flow) has been glued. It would be interesting to show the existence of an operation of "glueing a handle" (glueing a piece of geodesic flow) to any transitive Anosov flow.

Finally, let us remark that, taking $\left\{\eta_{j}\right\}_{j}$ such that $\bigcup_{j} \eta_{j}$ has $\ell$ connected components instead being connected, we obtain examples of transitive Anosov flows with $\ell$ pairwise non isotopic transverse tori.

\section{Proof of the theorem}

Recall that $\lambda_{g}$ (resp. $\nu_{g}$ ) is the largest eigenvalue of $A_{g}$ (resp. $B_{g}$ ). A direct computation shows that $\lambda_{35}=\nu_{25}$, hence we set

$$
\lambda=\lambda_{35}=\nu_{25} .
$$

The surface $\Sigma_{409}$ covers both $\Sigma_{35}$ and $\Sigma_{25}$. The multiplicity of a covering $\Sigma_{409} \stackrel{i}{\rightarrow} \Sigma_{35}$ is 12 , whereas the multiplicity of a covering $\Sigma_{409} \stackrel{j}{\rightarrow} \Sigma_{25}$ is 17 .

Let $\Phi_{35}: \pi_{1}\left(\Sigma_{35}\right) \rightarrow \mathrm{PL}_{+}^{\lambda}\left(\mathbf{S}^{1}\right)$ be the representation of corollary 1 and

$$
\Phi_{0}: \pi_{1}\left(\Sigma_{409}\right) \rightarrow \mathrm{PL}_{+}^{\lambda}\left(\mathbf{S}^{1}\right)
$$

its lift defined by $i: \Sigma_{409} \rightarrow \Sigma_{35}$. Clearly, $\Phi_{0}$ is topologically conjugate to $\Psi_{409}$. 
On the discrete Godbillon-Vey invariant and Dehn surgery on geodesic flows

Let $\Phi_{25}^{\prime}: \pi_{1}\left(\Sigma_{25}\right) \rightarrow \mathrm{PL}_{+}^{\lambda}\left(\mathbf{S}^{1}\right)$ be the representation of corollary $1^{\prime}$ and

$$
\Phi_{0}^{\prime}: \pi_{1}\left(\Sigma_{409}\right) \rightarrow \mathrm{PL}_{+}^{\lambda}\left(\mathbf{S}^{1}\right)
$$

its lift defined by $j: \Sigma_{409} \rightarrow \Sigma_{25}$. Also, $\Phi_{0}^{\prime}$ is topologically conjugate to $\Psi_{409}$ and hence to $\Phi_{0}$.

The discrete Godbillon-Vey invariant of $\Phi_{g}$ has been evaluated by Hashiguchi [Ha2]:

$$
\overline{\mathrm{GV}}\left(\Phi_{g}\right)=-(4 g+4)\left(\log \lambda_{g}\right)^{2}
$$

It follows that

$$
\overline{\mathrm{GV}}\left(\Phi_{0}\right)=12 \cdot(-4 \cdot 35-4)(\log \lambda)^{2}=-1728(\log \lambda)^{2}
$$

The discrete Godbillon-Vey invariant of $\Phi_{g}^{\prime}$ is an integral multiple of $(1 / 2)\left(\log \nu_{g}\right)^{2}$, and so

$$
\overline{\mathrm{GV}}\left(\Phi_{0}^{\prime}\right)=17 \cdot \frac{n}{2}(\log \lambda)^{2}, \quad n \in \mathbb{Z}
$$

Because $(1728 \cdot 2) / 17$ is not an integer, we necessarily have

$$
\overline{\mathrm{GV}}\left(\boldsymbol{\Phi}_{0}\right) \neq \overline{\mathrm{GV}}\left(\boldsymbol{\Phi}_{0}^{\prime}\right)
$$

and this completes the proof.

Remark. - The geodesic flow on $\Sigma_{g}$ is obtained from the suspension of $B_{g}: \pi^{2} \rightarrow \pi^{2}$ through $(1,1)$-Dehn surgeries along $4 g+2$ closed orbits of period 1; the result of Hashiguchi (see the comments at the end of the introduction of [Ha2]) suggests $\overline{\mathrm{GV}}\left(\Phi_{g}^{\prime}\right)=-(4 g+2)\left(\log \nu_{g}\right)^{2}$ and consequently $\overline{\mathrm{GV}}\left(\Phi_{0}^{\prime}\right)=-1734(\log \lambda)^{2}$.

\section{References}

[BL] Bonatti (C.) and Langevin (R.) - - Un exemple de flot d'Anosov transitif transverse à un tore et non conjugué à une suspension, Preprint (1992).

[Chr] Christy (J.) . - Intransitive Anosov flows, to appear on Memoirs AMS.

[Fri] Fried (D.) . - Transitive Anosov flows and pseudo-Anosov maps, Topology 22 (1983), pp. 299-303. 


\section{Marco Brunella}

[Ghy] Ghys (E.) .- Sur l'invariance topologique de la classe de Godbillon-Vey, Ann. Inst. Fourier 37, ${ }^{\circ} 4,(1987)$, pp. 59-76.

[GH] Ghys (E.) and SERgiescu (V.) .- Sur un groupe remarquable de difféomorphismes du cercle, Comm. Math. Helv. 62 (1987), pp. 185-239.

[Goo] Goodman (S.) .- Dehn surgery on Anosov flows, Geometric Dynamics, Springer Lecture Notes 1007 (1981), pp. 300-307.

[Ha1] Hashiguchi (N.) .- On the Anosov diffeomorphisms corresponding to geodesic flows on negatively curved closed surfaces, J. Fac. Sci. Univ. Tokyo 37 (1990), pp. 485-494.

[Ha2] Hashiguchi (N.) . - PL-representations of Anosov foliations, Ann. Inst. Fourier $42, \mathrm{n}^{\circ} 4$ (1992), pp. 937-965.

[HM] Hashiguchi (N.) and Minakawa (H.) .- Continuous variation of the discrete Godbillon-Vey invariant, J. Fac. Sci. Univ. Tokyo 39 (1992), pp. 271-278. 